rated as the three most highly relevant topics covered. Additionally, several individuals expressed a need for guidance and support in informing relatives and friends of their eating disorder, and one suggestion was that the final session be opened to such significant others for education. (A separate carers' group is already available within our service.) A common response from the majority of service users was that the group made them feel supported and less alone with their illness while awaiting treatment. We hope that this positive experience will help to perpetuate therapeutic engagement while they remain under the care of our service.

Improving the overall quality of a service user's experience when passing through the care pathway (as identified by Waller et al), with their direct involvement at all stages, from the booking of an initial appointment to a collaborative approach towards therapy, is essential in ensuring active engagement of service users with all psychiatric conditions. However, it is particularly important when attempting to support those whose illness has such strong egosyntonic qualities as anorexia in making a decision to pursue recovery.

1 Geller J, Zaitsoff S, Srikameswaran S. Tracking readiness and motivation for change in individuals with eating disorders over the course of treatment. CognitTher Res 2005; 29: 611-25.

2 Beato-Fernandez L, Rodriguez-CanoT. Eating disorders and stages of change: prognostic influence on eating psychopathology. In Eating
Disorders: New Research (ed. PI Swain): 245-63. Nova Science Publishing, 2006

3 Zeeck A, Hartmann A, Buchholz C. Drop outs from in-patient treatment of anorexia nervosa. Acta Psychiatr Scand 2005; 111: 29-37.

4 Guarda AS. Treatment of anorexia nervosa: insights and obstacles. Physiol Behav 2008; 94 : $113-20$

5 Waller G, Schmidt U, Treasure J, Murray K, Aleyna J, Emanuelli $F$, et al. Problems across care pathways in specialist adult eating disorder services. Psychiatr Bull 2009; 33: 26-9.

Sara L. Adshead Specialist Registrar, General Adult Psychiatry, Eating Disorder Service, Barberry Centre, 25 Vincent Drive, Edgbaston, Birmingham B15 2FG, email: Sara.adshead@bsmhft.nhs.uk

doi: 10.1192/pb.33.5.196b

\section{Doctors' human rights and the smoking ban}

Mental health trusts across the UK have implemented a smoke-free agenda that the National Health Service in England and Wales will be smoke-free by December 2006.' 'Smoke-free' means that smoking will not be permitted anywhere within hospital grounds, with no exceptions for staff or visitors and limited exemptions for certain patients, providing no one is subject to passive smoking as a result. ${ }^{2}$ Although this is acceptable in order to maintain government legislation on smoking in public places, one has to ask whether these rules should also apply to domiciliary visits?

If smoking by a service user, in their home, interferes with the doctor-patient consultation, then should the clinician be able to insist that the patient stop smoking, or is this a breach of their human rights? We have experience of domiciliary visits where the patient has insisted on smoking cigarettes during the consultation, despite requests to stop. As such visits may arise in the context of Mental Health Act assessments or urgent reviews, they may be perceived to be stressful by the patient, hence providing justification for smoking to relieve tension.

However, passive smoking is not only detrimental to the clinician's health. It may also have a negative impact on the therapeutic alliance, if the issue is confronted. And surely doctors' human rights are just as important as patients'?

1 Department of Health. Annual Report of the Chief Medical Officer 2003. Department of Health, 2004.

2 O'Gara C, Mclvor R. Smoke-free psychiatric services. Psychiatr Bull 2006; 30: 241-2.

*Asad Raffi Specialty Registrar, Mersey Care NHS Trust, Broadoak Unit, Thomas Drive, Liverpool, Merseyside L14 3PJ, email: asad.raffi@merseycare. nhs.uk, Jahangir Mahmood Specialty Registrar, Mersey Care NHS Trust

doi: 10.1192/pb.33.5.197

\title{
corrections
}

E-learning for psychiatrists. Psychiatric Bulletin, 33, 81-83.

The middle initial for Elizabeth Hare is 'H' not 'E'.

p. 83, col. 2, para. 1: Declaration of interest, the first sentence should read:
E.H.H. is the editor of CPD online and has spent more time on the internet than she would care to know.

p. 83, col. 2, para. 2: Acknowledgement, the sentence should read: With thanks to DrTom Conlon, former Senior
Lecturer. School of Education, University of Edinburgh, for his help with the planning of this article.

doi: 10.1192/pb.33.5.197a 